\title{
A new study of Lassa hemorrhagic fever model via Caputo-Fabrizio derivative
}

\author{
Abdullahi Yusuf ${ }^{1}$, Tukur Sulaiman ${ }^{2}$, and Pushpendra Kumar ${ }^{3}$ \\ ${ }^{1}$ Firat Universitesi \\ ${ }^{2}$ Biruni Universitesi \\ ${ }^{3}$ Central University of Punjab
}

May 6, 2020

\begin{abstract}
In the last three decades, we can see that an enthralling research topic that attracted the consideration of many researchers is mathematical modelling of biological systems. This paper is organised with the aim of getting some new simulations of lassa hemmorhagic fever; a deathly diseases in pregnant women via Caputo-Fabrizio fractional order derivative with the help of the Euler method. Lassa hemorrhagic fever is biocidal and epidemical disease, whose outbreaks were first conrmed in African countries. As compare to the Ebola virus this virus kills pregnant women's more. On 8 January, Lassa virus was detached in Vero cell cultures from a blood sample, which was ejective to be 12 days after the invasion of the illness. In this manuscript, important lemma and theorems are considered to exhibit the existence and uniqueness analysis, stability of proposed fractional approximation method. Results are provided to conrm the effectiveness of used approximation method by graphical simulations for different values of $\beta$.
\end{abstract}

\section{Hosted file}

Project Lassa fever.pdf available at https://authorea.com/users/144027/articles/447450-a-newstudy-of-lassa-hemorrhagic-fever-model-via-caputo-fabrizio-derivative 
figures/21/21-eps-converted-to.pdf 
figures/22/22-eps-converted-to.pdf 
figures/23/23-eps-converted-to.pdf 
figures/24/24-eps-converted-to.pdf 
figures/31/31-eps-converted-to.pdf 
figures/32/32-eps-converted-to.pdf 
figures/33/33-eps-converted-to.pdf 
figures/34/34-eps-converted-to.pdf 